\title{
Value of Ultrasound Examination of the Mastectomy Bed
}

\author{
Hesham E. El sheikh, Shorouk Z. Abdel Aziz, Rania M. Abdel Rahman
}

\begin{abstract}
Department of radiology, Benha faculty of medicine, Banha University, Egypt.

Correspondence to: Rania M. Abdel Rahman, Department of radiology, Benha faculty of medicine, Banha University, Egypt
\end{abstract}

Email:

raniaabdelrahman2021@gmail. com

Received: 19 August 2021

Accepted: 4 December 2021

\begin{abstract}
:
Background: Mastectomy mean surgical removal of all breast tissue and used as surgical treatment of breast cancer patients, follow up after surgery is very important for detecting abnormalities at the mastectomy. Aim: value of ultrasound (U/S) examination at mastectomy bed. Patients and methods: A prospective study included 100 female patients that underwent mastectomy and attended to Benha teaching hospital for follow up examination. 10 patients excluded (as 7 patient refused to participate , 3 patient not adherent to follow up),so the finally total 90 female patients were referred for, clinical examination following $U / S$ examination, and the final result depend on histopathological correlation or follow up 6monthes for at least one year. Results: 10 patients out of 90 had US suspicious feature and after biopsy, 8 patients of them proved as recurrence. Conclusion:
\end{abstract}

patients after mastectomy had risk for recurrent breast cancer so regular Follow-up U/S examination for early detecting abnormalities at the mastectomy site.

Keywords: Mastectomy, breast ultrasound (U/S), post-operative breast cancer (BC)

\section{Introduction:}

The surgical treatment of breast cancer aims to remove the breast lesions with or without remove lymphatic drainage and to reduce the possibility of local recurrence (LR) ( 1) . Type of surgery depend on type and stage of the tumour .there are two main options, breast-conserving surgery(BCS)and mastectomy (2). BCS mean only removal the tumour and surrounding normal tissue.

Mastectomy, is the surgical removal of all the breast tissue. (3)

Mastectomy used in women who had multicenteric breast cancer and/or large 
tumours. or extensive ductal carcinoma in situ. despite the Extensive resection of breast and lymphatic tissue, locoregional recurrences (LRRs) after mastectomy may occur .(4)

Due to postoperative changes, imaging evaluation of patients underwent mastectomy could be challenging. $\mathrm{U} / \mathrm{S}$ is the initial modality of choice for evaluating mastectomy bed, US allows observing subcutaneous fat, postsurgical scarring, and fibrosis. ( 5).

Possible complications or benign findings such as seromas or hematomas, fat necrosis, and fibrosis should also be assessed Nodular lesions on ultrasound suggest mainly recurrence. . (6)

\section{Patients and methods:}

Study population: This prospective study was carried out during period from March 2019 to December 2020 and included 90 female patients that underwent mastectomy due to breast cancer, all patients referred to radiology department of Benha teaching hospital from outpatient clinic of surgery department, all patient included in the study underwent clinical examination, clinical preoperative radiological data, operative details and US examination .
This study was approved by the Ethical Committee of Benha Faculty of Medicine, a written consent was taken from all subjects before enrolment of the study.

\section{Inclusion criteria:}

Female Patients underwent mastectomy and attended for follow up after operation .And in case of suspicious lesion, biopsy was requested or regular close up if refused biopsy.

\section{Exclusion criteria}

Patients who refused to participate in the study.

Patients not adherent for follow up .

\section{Methodology}

First clinical examination done.(at site of mastectomy bed, axilla, any symptoms, any felt or seen palpable lesions).

Clinical preoperative radiological data,(any imaging done before operation, pathological data preoperative)

Operative detail ( date of surgery, which type of mastectomy done).

U/S used for examination of the mastectomy site(used linear transducer 7.5-12 MHZ , about 4 cases used curved probe due to large mass finding and cant be measured by linear probe).

About 3 cases underwent mammogram adjuvant to US as 2of them were lumpectomy 
operation and the other was huge mass seen at mastectomy bed

\section{Procedure}

- The machine used was GE LOGIC P7, and On US guided biopsy the needle used $14 \mathrm{G}$ or $16 \mathrm{G}$ core biopsy needle, single or co-axial, with $10 \mathrm{~mm}$ or $20 \mathrm{~mm}$ cutting lengths.

- Patient lie supine (or anterior oblique) with arm abducted of ipsilateral area examined .

- US gel will be placed within the sleeve over the head of the probe.

- Examination of the mastectomy site and the axilla.

- This study will be included the results of mastectomy site and ipsilateral axillary fossa.

- Presence of a lesion the following should assess, solid or cystic ,the size, shape, border of the masses, color Doppler used (to determine vascularity), power Doppler used in one case, echo structures, and relationships to surrounding structures .

\section{The evaluation of the images}

- Patients with negative findings and probably benign nodules will be recommended for US follow-up at an interval of 6 months for more than 1year..
- Patients with suspicious for malignant nodules will be advised to undergo US-guided biopsy.

- Final diagnosis will be based on pathology results and clinical or sonographic follow-up for more than 12 months.

\section{Statistical analysis:}

The collected data analyzed using SPSS software qualitative data expressed as number and percentage while quantitative data expressed as mean \pm SD . Suitable statistical tests of significance calculated. $\boldsymbol{P}$ value less .than 0.05 significant.

\section{Results}

- The study included 90 female patients, ranging from 32 years to 77 years.

- U/S examination done for routine follow up in 57 patients, palpable lump in (11 patients, painful \& redness operative bed in 15 patients and suspected during clinical examination in 5 patients as ( Figure 1).

- As regards to type of operation, 3cases underwent partial mastectomy, 10 cases breast conservative surgery(BCS), 9cases simple mastectomy\&68 cases were modified radical mastectomy(MRM).38 patients out of 90 
patients were right sided operation, 52 patients were left sided (Table 1).

- The result of U/S examination was 40 patient were negative finding, 50 patients with positive finding(10 suspicious on U/S, 40 patients with benign looking lesions). (Table 2).

- The positive findings were fat necrosis (6), skin thickening \&edema (10), postoperative scar \&architecture distortion (13), seroma (11), and suspicious lesions in (10 cases). (Figure 2 )

- Location of suspicious lesions at mastectomy bed was, 5 cases at lateral side, 2 cases at central part, 3 cases at medial side.

- On U/S guided biopsy of 10 suspected lesions revealed 8 cases were malignant lesions, 2 cases benign findings(fat necrosis, fibrosis), and rate of local recurrence in the study was $8.8 \%$ (figure 3). And histological type, grade of malignant lesions as shown (Table 3)

- On follow up of positive benign looking lesions, no any lesions of them show progression or suspicious finding
- On follow up of negative finding one case developed suspicious lesion after $6 \mathrm{~m}$.

- The sensitivity, specificity, positive predictive value (PPV), and negative predictive value (NPV) of screening US were $90.9 \%, 98.0 \%, 52.6 \%$, and $99.8 \%$, respectively.(Table 4)

\section{Case presentation}

\section{Case No: 1(figure 4)}

Seventy two female patients underwent MRM for right breast IDC $4 \mathrm{y}$ ago prior to US, came for palpable swelling at medial site of surgical bed underwent mammography and US.

\section{Mammography\& US examination}

Show diffuse skin thickening, with underlying ill-defined dense mass lesion ,The mass lie at medial side of mastectomy scar as, Ill-defined suspicious solid mass lesion reaching deep muscle layer.

\section{Diagnosis based on pathology result}

Suggestive of malignancy corresponding to original tumour (IDC). 
Benha medical journal, vol. 39, special issue (radiology), 2022

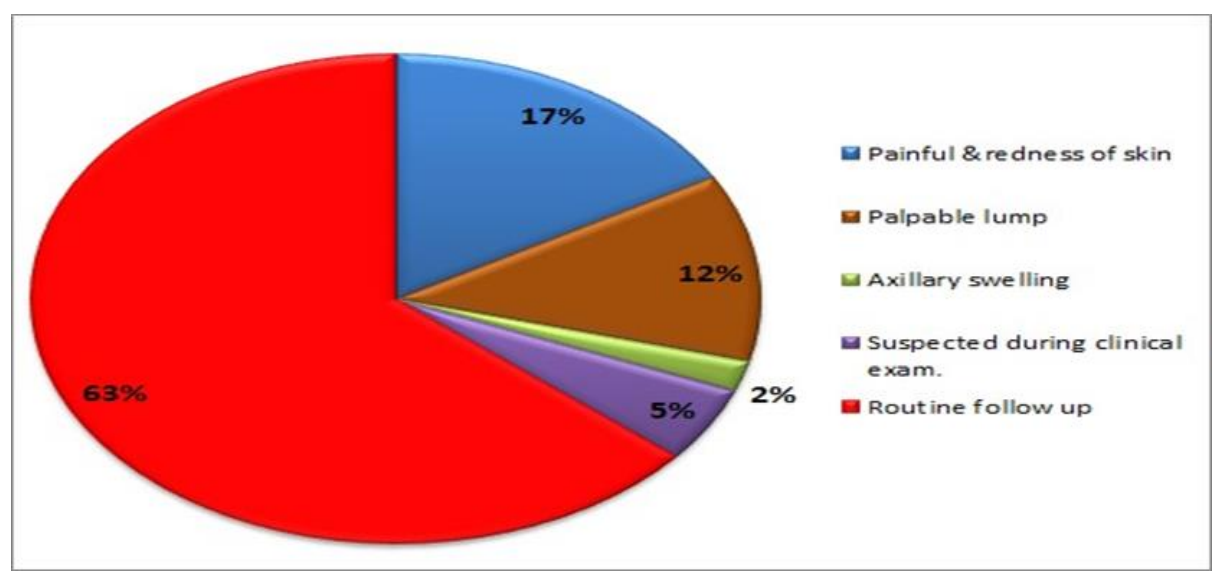

Figure 1: distribution of study group according to aim of $U / S$ examination

Table 1: show distribution of patients according to type \&side of operation

\begin{tabular}{cccc}
\hline Operation & Number & Side of operation & Number \\
\hline MRM & 68 & Right side & 38 \\
Simple mastectomy & 9 & & \\
Partial mastectomy & 3 & Left side & \\
BCS & 10 & & \\
Total & 90 & Total & 90 \\
\hline
\end{tabular}

Table 2: post -operative U/S finding at mastectomy bed.

\begin{tabular}{ccc}
\hline US finding & N & \% \\
\hline Negative & 40 & 44.44 \\
positive & 50 & 55.56 \\
Total & 90 & 100 \\
\hline
\end{tabular}

Table 3: show histological type \&grades of recurrent lesions

\begin{tabular}{cccc}
\hline Histological type & Number & Histological grade & Number \\
\hline Invasive ductal carcinoma & 6 & Grade I & $\mathbf{1}$ \\
Invasive lobular carcinoma & 2 & Grade II & $\mathbf{6}$ \\
& & Grade III & $\mathbf{1}$ \\
Total & 8 & Total & $\mathbf{8}$ \\
\hline
\end{tabular}




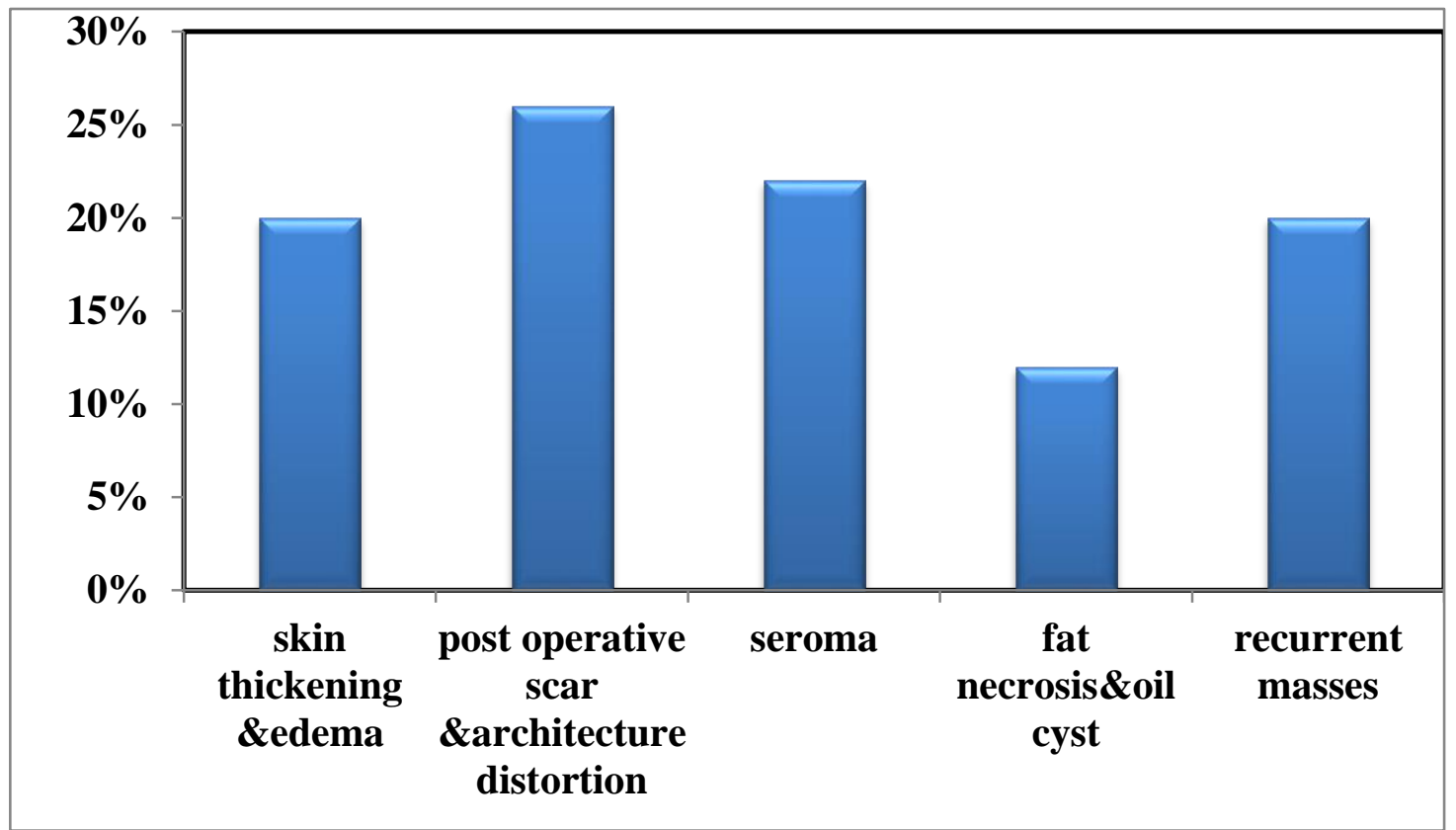

Figure 2: mass distribution of the studied group according to positive US find

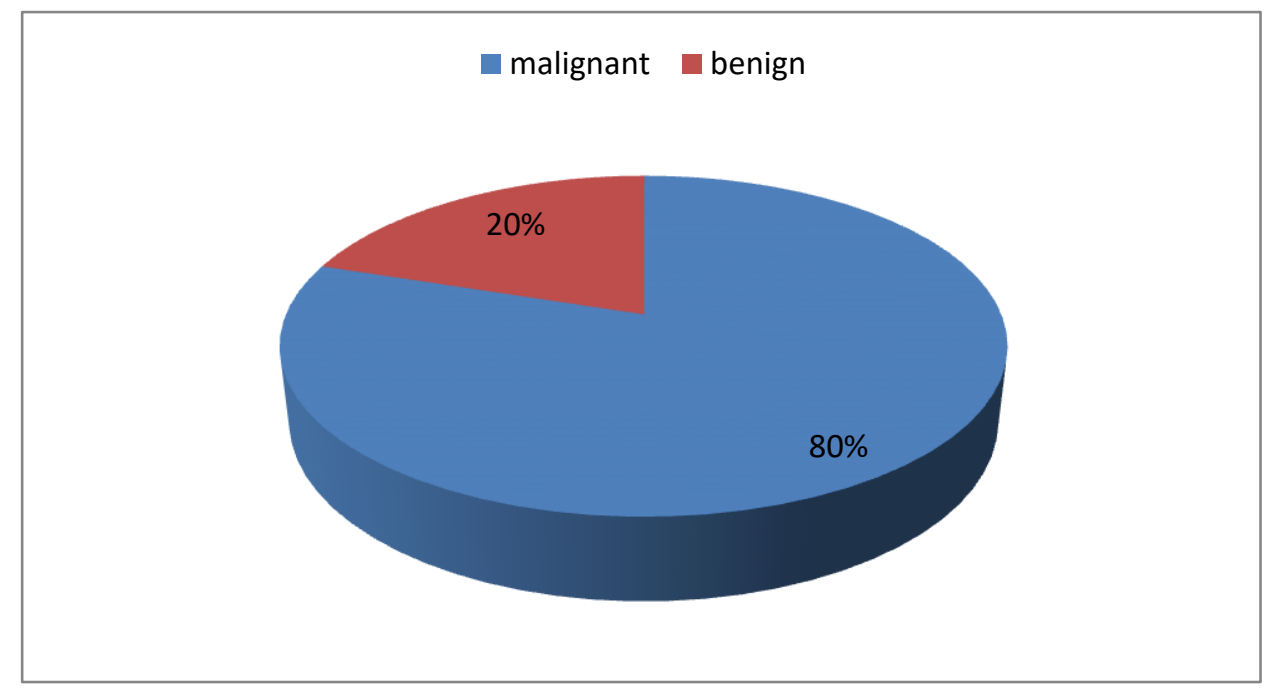

Figure 3: Distribution of the studied group according to the results of pathology. 
Table 4: show U/S final diagnosis.

\begin{tabular}{cccc}
\hline U/S findings & Benign lesions & Malignant lesions & Negative lesions \\
\hline Number of cases & 40 & 10 & 40 \\
On follow up/biopsy & $\begin{array}{c}\text { Stability and } \\
\text { regression of other } \\
\text { lesions,No suspicious } \\
\text { lesion developed } \\
\text { 100 }\end{array}$ & $\begin{array}{c}\text { pathologically as } \\
\text { malignant }\end{array}$ & $\begin{array}{c}\text { One cases developed } \\
\text { suspicious on follow } \\
\text { up, }\end{array}$ \\
$\begin{array}{c}\text { of U/S \% } \\
\text { Sensitivity of US }\end{array}$ & 94 & 97.5 \\
Specificity & 88.8 & \\
PPV & 95 & \\
NPV & & 97 & \\
\hline
\end{tabular}



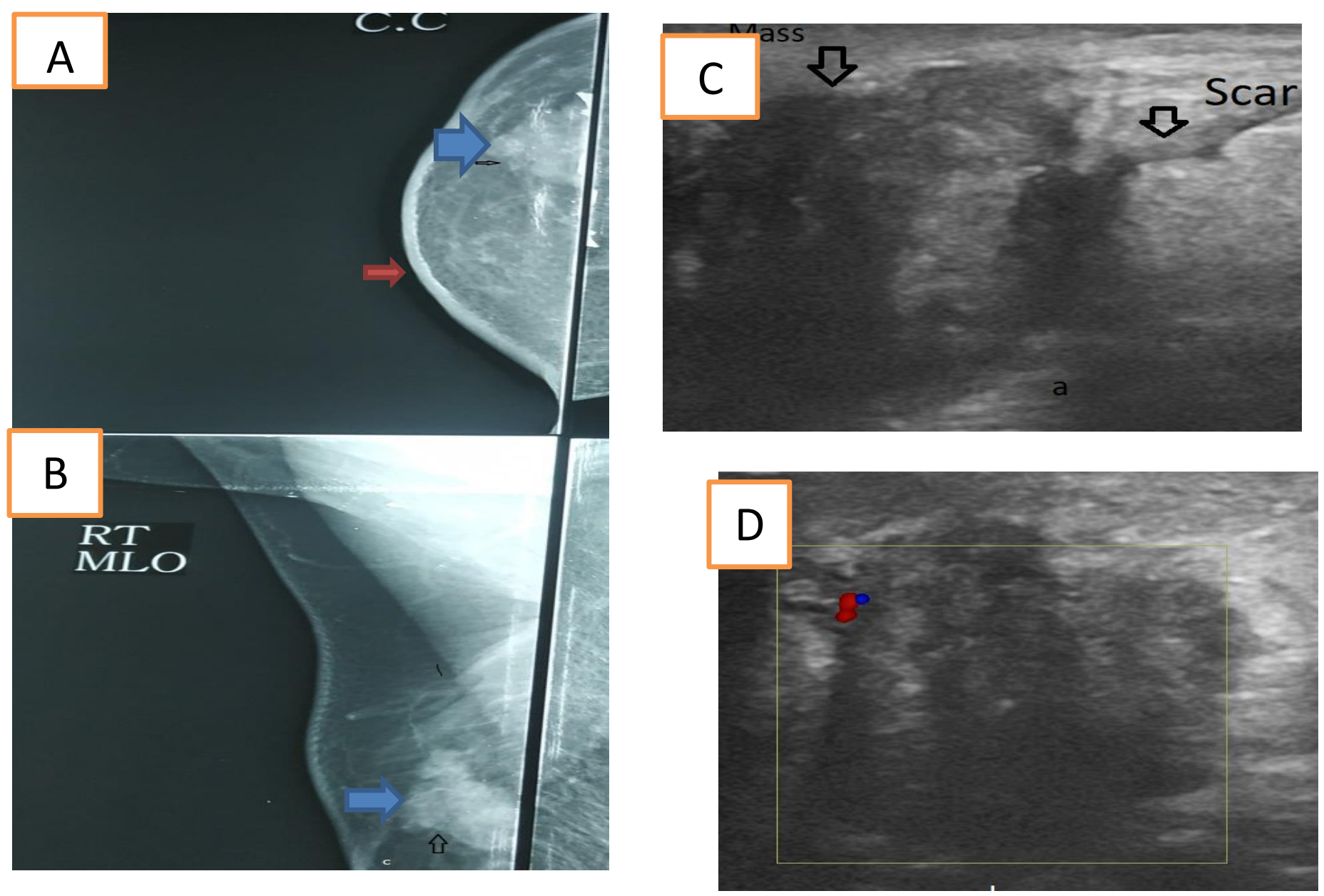

Figure 4: (A,B,C \& D) Mammo and U/S images of 72y old female patient underwent right MRM, presented by palpable lump at mastectomy site .show, CC\&MLO views of mammography, show irregular indistinct margin, hyper dense mass lesion(blue arrow at A,B) associated with diffuse skin thickening(red arrow at A) .at US image show, irregular, Ill-defined hypo echoic solid mass lesion(black arrow at C) and show posterior acoustic shadowing seen at medial end of scar tissue ( black arrow A), measuring $(2.1 \times 2.6 \mathrm{~cm})(\mathrm{b})$, show internal vascularity on Doppler examination(D).

\section{Discussion}

The purpose of the study was to evaluate US findings in the patients who underwent mastectomy, and came for follow up. Mastectomy still plays an important role in $\mathrm{BC}$ surgical treatment.

Several previous studies had included the value of U/S surveillance for $\mathrm{BC}$ patients after surgery. One study (7) investigated the efficacy of locoregional U/S examinations for the detection of recurrence in asymptomatic patients who underwent mastectomy and the impact of on prognosis. It found that LUE was helpful for the early detection in $\mathrm{BC}$ recurrence in patients with $\mathrm{BC}$ after mastectomy, and it may result in a better prognosis for patients. The other study (8) assessed the diagnostic performance of ultrasound examinations for the malignant lesion detection in patients with postoperative $\mathrm{BC}$, and evaluated its clinical value in such kinds of patients It concluded 
that postoperative ultrasound examinations for detection of malignant lesions in patients with BC had a high sensitivity Thus, it can be used as a predictor of distant metastasis in patients received mastectomy, but not for patients with breast conservation surgery..

The results of our study are consistent with the previous studies $(\mathbf{7 , 8})$. We evaluated the clinical usefulness of breast U/S examination in patients after BC surgery. We found that US can be used for the detection of the benign \& malignant lesions(in case of malignant US guided biopsy done for final pathological diagnosis) in patients of BC after surgery with high sensitivity\& specificity.

In the current study included 90 female patients, the age group ranged between (26$62 y)$ with mean age of about51.7 years , Underwent different type of mastectomy, 9simple mastectomy, 10 BCS,3 partial mastectomy \&78MRM .52 of them underwent mastectomy operation at the left side and the rest (38 patient) at right side .

Which is similar to result done by Radhika et al (2016) where his study include 183 patients aged between 22 and 78 years with a mean age of 53 years(5) .smaller mean of age was documented to cases studied by Liu et al., (2017) being 45.9(9)
Yilmaz M. et al where the former included 27 women , 16 underwent left mastectomy \& 11 right mastectomy for breast cancer.(10)

Havenge et al.,(1992) 14 of the patients underwent radical mastectomy and 86of the patients underwent modified radical mastectomy.(11)

The results of US examination reveal 40 patient were negative finding, and 40 patients with benign\& 10suspicious finding post-operative. In the study done by Lee etal ., in 2013 (12)included 468 patients who had undergone mastectomy, U/S examinations were as follows: negative in 389 (83.1\%), probably benign nodules in 60 (12.8\%), and suspicious for malignant nodules in 19 (4.1\%), Also in the study done by Sridharan etal.,in 2016(5) On U/S 175 cases were normal, three cases were benign and five cases indeterminate .

In our study, The benign US findings were 6 cases fat necrosis \&oil cyst,11 patients seromas, 10 skin thickening and oedema \&13 patients post-operative scar\& architecture distortion . which was similar to study done by Mehrotra s. et al(13)the former study include 35 patients had skin thickening at the post-operative site , 32 patients have seroma underlying the 
postoperative site. 18 patients had postoperative architectural distortion , 9 patients had presented with fat necrosis and dystrophic calcification. 4patients presented with oil cysts.

US finding of 10 suspicious lesions 5 patient of them located at lateral side of mastectomy bed, 2 patients at central part of surgical bed $\& 3$ patients at medial side, which is similar to study done by previously (11) where the location of recurrent disease at mastectomy site were lateral 12 , medial 11 , central 7 patients.

The present study included 10 Suspicious malignant lesion at US examination, 8 of lesions seen at mastectomy site (chest wall) 2lesions at ipsilateral axilla, which similar to result at study done by Kim et al., in 2010(7) in which No. of ultrasound positive lesions at mastectomy bed 10\&at ipsilateral axilla 6

The rate of local recurrence in this study was $(8.8 \%)$ diagnosis in 8 patient out of 10 patient that had suspicious lesions observed in US examinations the other two lesions were, fat necrosis \&fibrosis which was higher than study done by Lee etal.,2013(12) Cancer detection rates were $2.1 \%$. in Yilmaz et al .2007 Local recurrence was diagnosed in 9 out of 11 cases(3.9\%) that had suspicious lesions observed in US examinations

. On the follow-up of 40 patients who had negative findings, there was 1 falsenegative case. The patient developed a palpable recurrent malignancy at the mastectomy site at 6 months after negative findings on US as the study done by Lee et al., 2012.

Our study reveal result of sensitivity , specificity, PPV, NPV\& accuracy of US at mastectomy site was $88.8 \%, 95.1 \%, 80 \%$, $97.5 \% \& 94 \%$ respectively. Higher results were at study done Liu et al.,(2017) The sensitivity(100\%), $\quad$ specificity $(99.7 \%)$, accuracy $(99.7 \%)$, positive predictive value $(36 \%)$,

Our study has some limitation. The first one, the sample size was small for a screening study so we recommended further study should include large sample size. The second, The study was conducted at a single institutional with only one radiologist., so we recommended further study with multicenteric institutional. The third, among positive US examinations, and not underwent biopsy, we followed US stability for more than one year, and more number of patients not adherent to follow up, such recurrence might missed in this study, 


\section{Conclusion}

U/S was helpful in assessment of mastectomy bed, with high accuracy (94\%). As it was able to detect post-operative changes, (benign\& malignant lesions) and correlated this changes were correlated with clinical and pathology

\section{References:}

1) Kaidar-Person O., Boersma L.,Poortmans P. Residual Glandular Breast Tissue After Mastectomy. Residual Glandular Breast Tissue after Mastectomy A Systematic Review, Annals surgery of oncology. 2020(27)2288-6229.

2) Lam D L., Houssami N, and Lee JM., Imaging Surveillance After Primary Breast Cancer Treatment, AJR Am J Roentgenol. 2017 ; 208(3): 676-686.

3) Jones C\& Lancaster R, Evolution of Operative Technique for Mastectomy, Surg Clin N Am 2018 (98) 835-844.

4) Sharma G, Dave R, Sanadya J, Sharma, and K. K. Sharma. 2010. Various Types and Management of Breast Cancer: An Overview. Journal of Advanced Pharmaceutical Technology \& Research 1(2):109-26.

5) Sridharan R, Baherin ,Abdullah R, The follow-up of post-mastectomy patients: Should the ipsilateral side be assessed with both mammogram and ultrasound? Med 2016 ,71 No 5 .

6) Rodrigo SP and Morris EA, Imaging Findings After Surgery, breast cancer.2017;3-319,6-21.
7) Kim HJ, Kwak JY, Choi JW, Impact of US surveillance on detection of clinically occult locoregional recurrence after mastectomy for breast cancer. Ann Surg Oncol 2010;17:2670-6.

8) Suh YJ, Kim MJ, Kim EK, Moon HJ, Kim SI, Park BW, Value of ultrasound for postoperative surveillance of asian patients with history of breast cancer surgery: a single-center stud.Ann Surg Oncol. 2013 (11):3461-8.

9) Liu YQ, Dong JN, Meng QX, Ultrasound for postoperative surveillance after mastectomy in patients with breast cancer A retrospective study. Medicine 2017, 96:52.

10) Yilmaz MH, Esen G, Ayarcan $Y$, Aydogan F, Özgüroglu M, Demir G, et al. The role of US and MR imaging in detecting local chest wall tumor recurrence after mastectomy. Diagnostic and Interventional Radiology. 2007 Mar 1;13(1):13.

11) Havenga K, Welvaart K\& Hermans J, Significance of local recurrence after mastectomy for breast cancer ,Surgical Oncology 1992; 1: 363-369.

12) Lee JH, Kim EK, Young J, US screening for detection of nonpalpable locoregional recurrence after mastectomy ,European Journal of Radiology 2013 (82) 485- 489.

13) Mehrotra S, Syed A, Ravi B, Spectrum of Mammography and Ultrasonography Findings in Imaging of Post-Surgical Breast: Many Facets, International Journal of Contemporary Medicine Surgery and Radiology. 2019;4(3)200-206.,

To cite this article: Hesham E. El sheikh, Shorouk Z. Abdel Aziz, Rania M. Abdel Rahman. Value of Ultrasound Examination of the Mastectomy Bed. BMFJ 2022; 39 (Radiology): 244-254. DOI: $10.21608 / \mathrm{bmfi} .2021 .91615 .1460$ 